\title{
Polychromatic nanofocusing of surface plasmon polaritons
}

\author{
Wei Liu, Dragomir N. Neshev, Andrey E. Miroshnichenko, Ilya V. Shadrivov, and Yuri S. Kivshar \\ Nonlinear Physics Centre, Centre for Ultrahigh-bandwidth Devices for Optical Systems (CUDOS), Research School of Physics and \\ Engineering, Australian National University, Canberra, ACT 0200, Australia
}

(Received 14 November 2010; published 17 February 2011)

\begin{abstract}
We introduce the concept of polychromatic plasmonics and suggest a broadband plasmonic lens for nanofocusing of surface plasmon polaritons. The lens employs a parabolically modulated metal-dielectric-metal structure. This plasmonic lens has a bandwidth of more than an optical octave, thus opening new opportunities for broadband plasmonic applications.
\end{abstract}

DOI: 10.1103/PhysRevB.83.073404

PACS number(s): 42.25.Bs, 78.67.Pt

The field of plasmonics experiences an explosive growth recently due to the ability of plasmonic components to confine light down to the nanoscale. Various plasmonic structures with miniaturization scales comparable to those of modern semiconductor electronics have been designed to realize different types of light waveguiding and control. ${ }^{1-7}$ Despite the boom in research one can identify two major challenges for further applications of plasmonic devices: high propagation loss and strong wavelength dispersion. Different approaches to combat optical losses in metals have been explored, including incorporation of gain ${ }^{8}$ or nanofocusing. ${ }^{9,10}$ However, the concept of broadband control and nanofocusing of polychromatic surface plasmon polaritons (SPPs) remains practically unattainable. At the same time, the requirement to manipulate ultrashort on-chip optical pulses at the nanoscale makes searching for approaches to solve this problem more urgent.

In this Brief Report, we introduce the concept of polychromatic plasmonics, of which the wavelength-independent optical potential is the key feature. We utilize quadratic modulation of the thickness of the dielectric layer in a metal-dielectricmetal (MDM) structure [Fig. 1(b)] to produce a parabolic optical potential which is wavelength independent. We develop analytical descriptions and employ numerical simulations to show its capability of three-dimensional subwavelength manipulations, including nanofocusing, self-collimation, and optical pendulum effect. The nanofocusing is demonstrated over a bandwidth exceeding an optical octave $(>500 \mathrm{~nm})$, thus allowing for polychromatic plasmon focusing, which addresses simultaneously the two big challenges mentioned earlier.

The concept of polychromatic light propagation is well developed for dielectric structures, of which the polychromatic dynamic localization is a noticeable example. ${ }^{11}$ In these structures, curved waveguides are employed to produce an effective wavelength-invariant optical potential to compensate for the dispersion.

In plasmonics, the engineering of the optical potentials for SPPs has been a key concept for designing plasmonic lenses and other elements. 5,6,12 Two kinds of structures with transverse ${ }^{4,7}$ or longitudinally varied effective index ${ }^{9,13}$ are mainly used. In the structures with transverse index modulation, the plasmonic potentials are usually heavily wavelength dependent. ${ }^{4-7}$ On the other hand, in longitudinally modulated structures it is shown in Ref. 13 that light of different wavelengths can be focused at the edge, however, light is fully localized there and could not propagate beyond. Although light could be prevented from being fully localized by opening up the taper, ${ }^{14}$ truncating the tips of the tapers would introduce standing waves, ${ }^{15}$ which are highly wavelength dependent and thus not suitable for pulse signal processing. Recently, a variation of a plasmonic lens was shown to focus broadband light based on diffraction. ${ }^{16}$ However, the focusing was obtained for light in free space rather than for propagating SPPs, thus with low focusing resolution. The focusing of polychromatic SPPs still remains an unsolved challenge.

To address this challenge, first we consider a flat MDM waveguide shown in Fig. 1(a). We only consider the symmetric mode of the structure (with respect to the magnetic field distribution) because it is primarily excited by simple end-fire coupling, while the antisymmetric mode experiences a cutoff for the parameters of our work. ${ }^{2}$ The dielectric is chosen to be silica glass with permittivity $\epsilon=2.25$, and the metal is silver for which we use the Drude model, $\varepsilon_{\mathrm{m}}=1-\omega_{p}^{2} /\left(\omega^{2}+\right.$ $\left.i \omega \omega_{c}\right)$, where $\omega, \omega_{p}$, and $\omega_{c}$ are the angular frequency, plasma frequency, and collision frequency, respectively. This model is proven to be a good approximation for noble metals, including silver in the spectral range above $500 \mathrm{~nm}$. For silver we take $\omega_{p}=1.37 \times 10^{16} \mathrm{rad} / \mathrm{s}$ and $\omega_{c} / \omega_{p}=0.002$. Figure 1(c) shows the real part of the mode index, $n_{\text {eff }}$, versus the inverse thickness of the dielectric for three different wavelengths. The dependence is linear, consistent with the theoretical approximation: ${ }^{17,18}$

$$
n_{\mathrm{eff}}=a / h+b,
$$

where both $a$ and $b$ are complex parameters and could be extracted from data fitting. Most importantly, the slope $a$ of these curves is practically wavelength independent, which allows for the design of broadband optical potential and polychromatic plasmon propagation.

Using the effective refractive index in Eq. (1), it is possible to construct a parabolic optical potential ${ }^{19}$ for SPPs. This is achieved by a MDM waveguide with one flat and one parabolically curved surface as shown in Fig. 1(b). The thickness of the dielectric is $h(x)=h_{0}+x^{2} / 2 R_{0}$, with $R_{0}$ as an effective radius $\left(R_{0} \gg h_{0},|x|\right)$. In this waveguide, we obtain a parabolic optical potential under the condition of $x^{2} \ll 2 h_{0} R_{0}$ :

$$
\varepsilon_{\mathrm{eff}}=n_{\mathrm{eff}}^{2}(x) \approx n_{0}^{2}\left(1-\Omega^{2} x^{2}\right),
$$


(a)

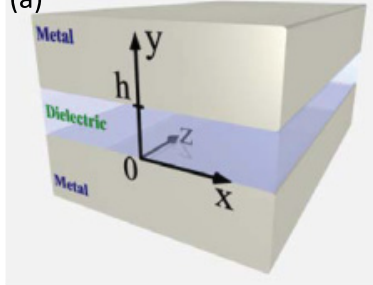

(b)

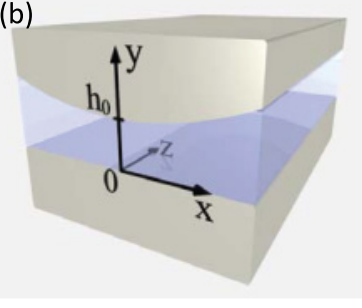

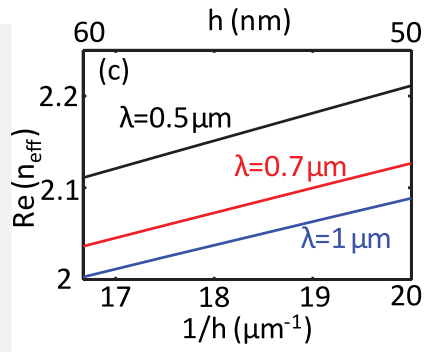

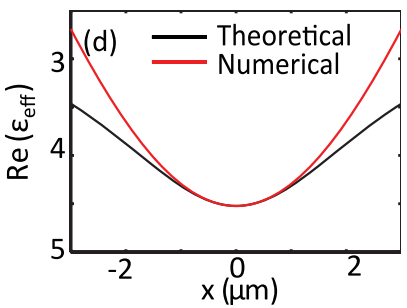

FIG. 1. (Color online) (a) Flat and (b) parabolically modulated MDM. (c) Real part of the symmetric mode index of the flat structure versus $1 / h$ for various values of free-space wavelengths. (d) Real part of the effective permittivity (optical potential) of the structure (b), obtained using Eq. (2) (black) and numerically (red).

where $n_{0}=a / h_{0}+b$, and $\Omega=\sqrt{a /\left(n_{0} R_{0} h_{0}^{2}\right)}$ is the focusing strength. Figure 1(d) shows the results calculated by Eq. (2) and numerically for $h_{0}=50 \mathrm{~nm}, \lambda=0.7 \mu \mathrm{m}$, and $R_{0}=100 \mu \mathrm{m}$.

Using the effective index method for the structure shown in Fig. 1(b), we can express the vertical electric field as $E_{y}(x, y, z)=A(x) B(x, y) \exp (i \beta z) .{ }^{18,20}$ When $|x| \ll R_{0}, A(x)$ and $B(x, y)$ could be decoupled. ${ }^{18}$ The expression for $B(x, y)$ could be found in Refs. 2 and 18 while the equation for $A(x)$ is ${ }^{19,21}$

$$
\frac{d^{2} A(x)}{d x^{2}}+\left[\zeta^{2}(x)-\beta^{2}\right] A(x)=0,
$$

where $\zeta(x)=k n_{\text {eff }}(x)$ and $\zeta_{0}=k n_{0}, k=2 \pi / \lambda$. This is a harmonic oscillator equation with eigenmodes $A_{m}(x)=$ $\left(\sqrt{\pi} \eta_{0} 2^{m} m !\right)^{-\frac{1}{2}} H_{m}\left(x / \eta_{0}\right) \exp \left(-x^{2} / 2 \eta_{0}^{2}\right),{ }^{19,21}$ where $\eta_{0}=$ $\left(\zeta_{0} \Omega\right)^{-\frac{1}{2}}$ is the characteristic width of the plasmonic waveguide, $H_{m}$ is the Hermite polynomial, and the effective indices of different modes, under the paraxial approximation, are $n_{\text {eff }}(m)=\left[\zeta_{0}-\frac{1}{2}(2 m+1) \Omega\right] / k$.

We also calculate the eigenmodes of this structure using commercial Mode Solutions (MS) software (LUMERICAL) with the parameters $x \in[-3,3] \mu \mathrm{m}, y \in[-1,1.05] \mu \mathrm{m}, R_{0}=$ $100 \mu \mathrm{m}, h_{0}=50 \mathrm{~nm}$, and $\lambda=0.7 \mu \mathrm{m}$. The results of our analytical theory [Eq. (3)] and the MS calculations are summarized in Fig. 2. Figures 2(a) and 2(b) shows the real and imaginary parts of $n_{\text {eff }}$ for different modes with the theoretical field distribution of $\left|A_{m}(x) B(x, y)\right|$ [Figs. 2(c)-2(e)] and numerically calculated total field $\left|E_{y}\right|$ [Figs. 2(f)-2(h)], which show a good agreement for low-order modes. For higher-order modes, a larger discrepancy appears in terms of both effective index and field distribution. This is consistent with the results in Fig. 1(d), as the higher-order modes spread out to the larger $x$ values, where the assumption $x^{2} \ll 2 h_{0} R_{0}$ does not strictly hold and the potential is not exactly parabolic.

Next we study the SPP propagation in the structure. An incident beam could excite modes of different orders, which
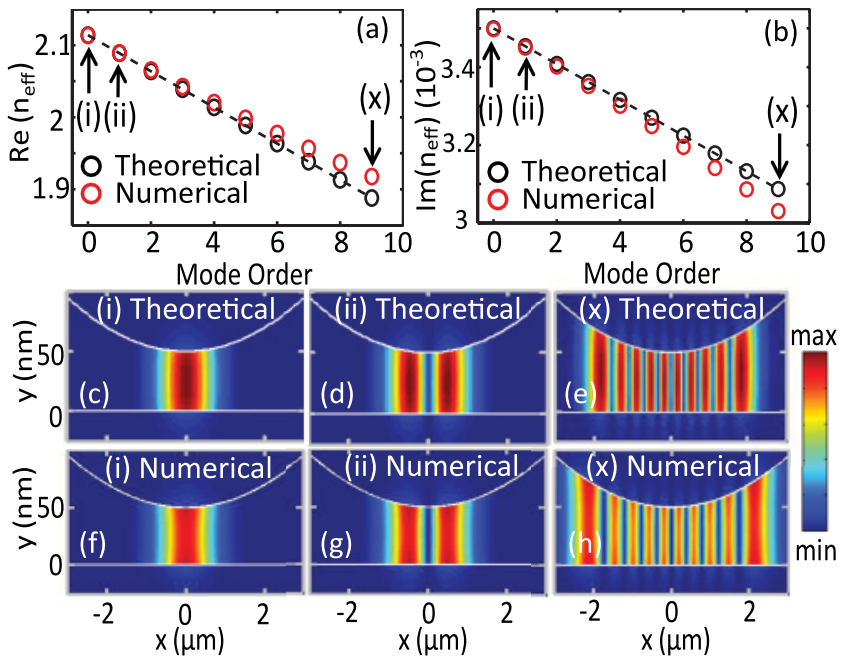

FIG. 2. (Color online) (a) Real and (b) imaginary parts of $n_{\text {eff }}$ for different-order modes. Theoretical (black circles) and numerical (red circles) results are shown. (c)-(h) Transverse mode profiles of the fundamental-, second-, and tenth-order modes. (c)-(e) Theoretically and (f)-(h) numerically calculated mode profiles $\left(\left|E_{y}\right|\right), \lambda=0.7 \mu \mathrm{m}$.

will then interfere with one another, producing different intensity patterns. As a result of this interference, a range of SPP beam manipulations is possible, including focusing, self-collimation, and optical pendulum effect. ${ }^{19,21}$ When $A(x)$ can be decoupled from $B(x, y)$ and $B(x, y)$ changes slowly with $x$, the input beam expansions along $x$ and $y$ inside the structure are independent. Considering that the beam distribution along $y$ can be fully characterized by $B(x, y)$, we study only the beam dynamics in the $x-z$ plane. The beam could be expanded into a complete set of orthogonal $\operatorname{modes}^{21} C(x, z)=\sum_{m=0}^{\infty} a_{m} A_{m}(x) \exp \left(i \beta_{m} z\right)$, where $a_{m}$ is the expansion coefficient of the $m$-order mode. If the initial beam has a Gaussian distribution $C(x, 0)=\pi^{-\frac{1}{4}} w_{0}^{-\frac{1}{2}} \exp [-(x-$ $\left.\left.x_{0}\right)^{2} / 2 w_{0}^{2}\right]$, where $w_{0}$ is the beam width, the beam inside the structure, under paraxial approximation, is ${ }^{19,21}$

$$
\begin{aligned}
C(x, z)= & \pi^{-\frac{1}{4}} q(z)^{-\frac{1}{2}} \exp \left\{-\frac{1}{2 \eta^{2}}\left[x-x_{0} \cos (\Omega z)\right]^{2}\right\} \\
& \times \exp \left[i\left(k_{0} z+\frac{1}{2} k_{0} \rho^{-1} x^{2}\right)\right] P\left(x_{0}\right),
\end{aligned}
$$

where $\eta^{2}=|q(z)|^{2}, \quad \rho^{-1}=\eta^{-1} d \eta / d z, \quad P\left(x_{0}\right)=\exp \left\{i\left(\eta_{0} /\right.\right.$ $\left.\left.w_{0}\right)^{2}\left[x x_{0} \sin (\Omega z)-\frac{1}{4} x_{0}^{2} \sin (2 \Omega z)\right]\right\}$, and $q(z)=w_{0} \cos \left(\Omega_{z}\right)+$ $i\left(\eta_{0}^{2} / w_{0}\right) \sin (\Omega z)$. In the lossless case with $\omega_{c}=0$, the beam intensity can be expressed as $|C(x, z)|=$ $\pi^{-\frac{1}{4}} \eta^{-\frac{1}{2}} \exp \left\{-\left[x-x_{0} \cos (\Omega z)\right]^{2} / 2 \eta^{2}\right\}$ with dynamic beam width varying along propagation:

$$
\eta=\sqrt{w_{0}^{2} \cos ^{2}(\Omega z)+\eta_{0}^{4} / w_{0}^{2} \sin ^{2}(\Omega z)} .
$$

The plasmon polariton beam is thus oscillating periodically along $z$. It is easy to prove that the maximum and minimum dynamic beam widths are constrained by ${ }^{19} \eta_{\max } \eta_{\min }=\eta_{0}^{2}$, indicating that the beam is trapped in the parabolic optical potential.

To confirm our theoretical analysis, we preform finitedifference time-domain (FDTD) simulations (LUMERICAL). 


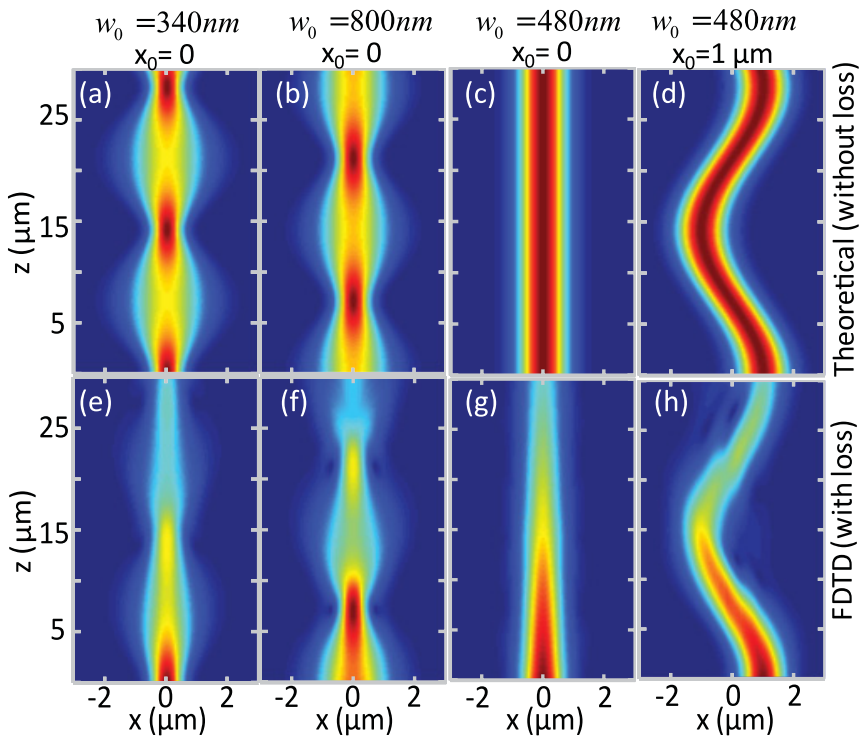

FIG. 3. (Color online) (a)-(d) Theoretical field distribution without loss and (e)-(h) FDTD simulations with loss (shown $|E|$ ) in the $x-z$ plane in the middle of the gap $\left(y=h_{0} / 2=25 \mathrm{~nm}\right)$. Plots (a), (b), (e), and (f) show typical effects of self-imaging with nanofocusing; (c) and (g) self-collimation; and (d) and (h) optical pendulum effect. The corresponding characteristic beam width is $\eta_{0} \approx 480 \mathrm{~nm}, \lambda=0.7 \mu \mathrm{m}$.

All initial beams are $y$ polarized with transverse Gaussian distributions to guarantee the excitation of SPP modes. The structure parameters are the same as in the MS simulation, with $z \in[0,30] \mu \mathrm{m}$ and perfectly matched layers at the boundaries. The analytical results without loss and the numerical results with loss are presented in Fig. 3. The data are shown for the $x-z$ plane in the middle of the gap, $y=h_{0} / 2=25 \mathrm{~nm}$.

For an on-axis input beam $\left(x_{0}=0\right)$ [Figs. 3(a), 3(b), 3(e), and $3(\mathrm{f})]$, one can observe periodic beam focusing with a period of

$$
F=\pi / \Omega=\pi \sqrt{R_{0} h_{0}\left(1+h_{0} b / a\right)} .
$$

The points of maximum intensity correspond to the focusing when all excited modes interfere constructively. There is an initial phase shift depending on the ratio of the incident beam width and the characteristic width of the plasmonic waveguide $w_{0} / \eta_{0}$. When these two widths match $w_{0}=\eta_{0}$, one can observe the self-collimation effect-diffractionless propagation of the beam [see Figs. 3(c) and 3(g)]. For an off-axis input beam $\left(x_{0} \neq 0\right)$ there are transverse oscillations [see Figs. 3(d) and 3(h)], and the plasmon polariton beam propagation exhibits an analog of optical pendulum behavior in a parabolic potential. The comparison with FDTD results suggests that losses do not affect the beam propagation except for attenuation along the $z$ direction.

Equation (6) has some important implications for propagation of polychromatic SPPs beams. As $a$ is practically wavelength independent while $b$ varies slowly [Fig. 1(c)], the focusing strengths $\Omega$ and the oscillation period $F$ are nearly the same for different wavelengths. For example, in Fig. 4(a) we show both the theoretical (solid line) and FDTD (circles) results for an input beam with $w_{0}=800 \mathrm{~nm}>\eta_{0}$ for three different thicknesses $h_{0}$ in the wide wavelength

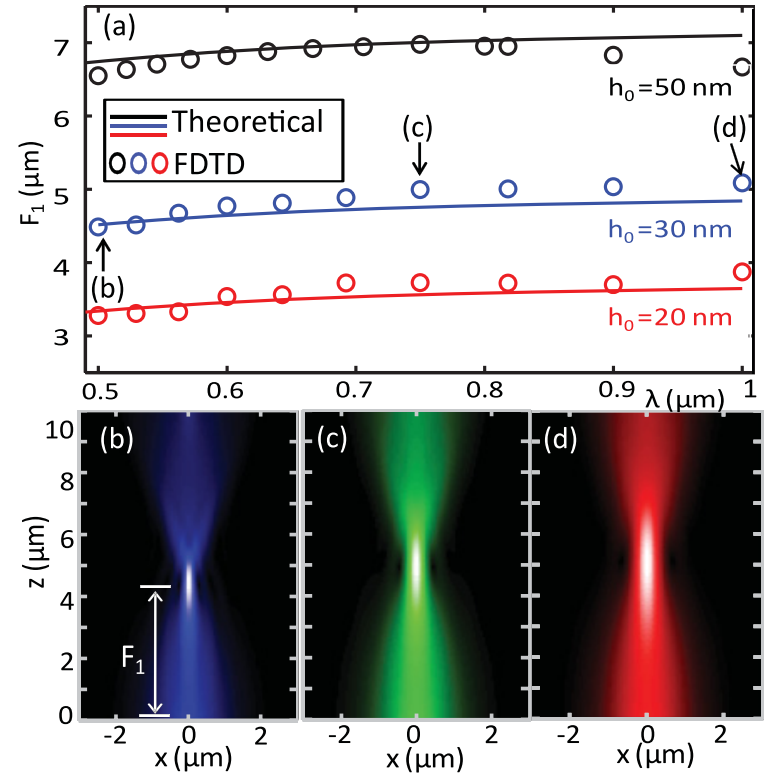

FIG. 4. (Color online) (a) Focusing position $z=F_{1}$ for wavelengths in the range $0.5-1.0 \mu \mathrm{m}$ at $h_{0}$ values of 20,30 , and $50 \mathrm{~nm}$. Solid lines, theoretical results; circles, FDTD simulations. (b)-(d) SPPs propagation for the three wavelengths marked in (a).

range $0.5-1.0 \mu \mathrm{m}$. The different color beams focus at the planes of $z=F_{1}=F / 2$. The dispersionless characteristic of $F$ enables broadband SPPs focusing and distinguishes our plasmonic structure from other highly wavelength-dependent structures. ${ }^{4,7}$ In Figs. 4(b)-4(d) we show the field distribution $|E|$ on the $x-z$ plane of $y=h_{0} / 2=15 \mathrm{~nm}$ for the three points marked in Fig. 4(a) at $h_{0}=30 \mathrm{~nm}$. False colors are used to indicate schematically the three different wavelengths. It is important to note that the focal planes of all spectral components coincide within $500 \mathrm{~nm}$ (less than one wavelength deviation in the spectral range $0.5-1.0 \mu \mathrm{m}$ ) and are practically indistinguishable. Note that the focal depth is in the range of 2-3 $\mu \mathrm{m}$ for the different spectral components. Figure 4 proves
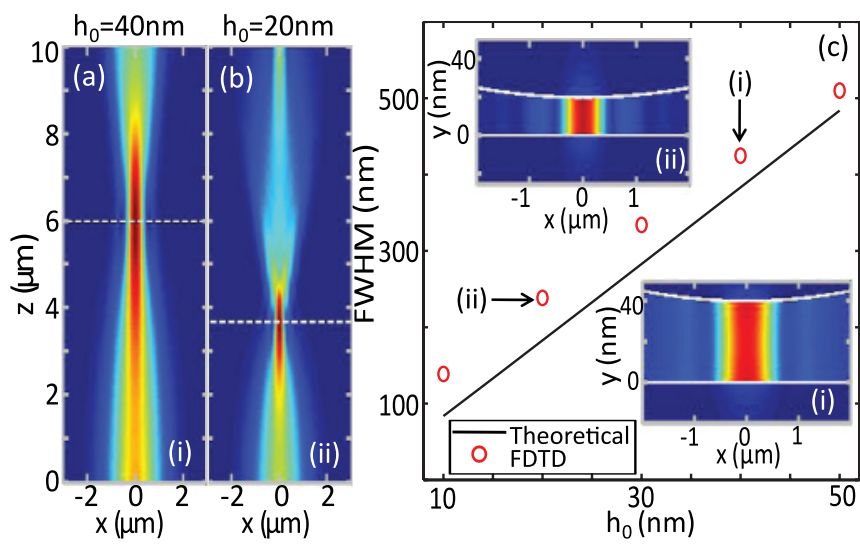

FIG. 5. (Color online) (a) and (b) FDTD results for the field distribution, $|E|$, in the $x-z$ plane $y=h_{0} / 2(h=40$ and $20 \mathrm{~nm})$ and $\lambda=0.7 \mu \mathrm{m}$. White dashed lines indicate the focal plane. (c) FWHM in the focal plane versus $h_{0}$. Marked points correspond to the cases (a) and (b), respectively. (Insets) Field distributions $|E|$ in the two marked focal planes. 
that this structure acts as a lens for broadband polychromatic light focusing, a functionality that has never before been discussed in other plasmonic structures. From Fig. 4 and Eq. (6), it is clear that one can control the position of the focusing points by varying the thicknesses $h_{0}$ and/or the wire curvature $R_{0}$, which enables wide reconfigurability of our structure.

Finally, we investigate the focusing resolution of this structure, which is characterized by the full width at half maximum (FWHM) along the $x$ direction at the focal plane. (Along the vertical direction, the resolution can be approximately characterized by the thickness of the dielectric layer as shown in the insets of Fig. 5.) We define the wavelength of the SPPs, $\lambda_{\text {spp }}=\lambda / n_{\text {eff }}$, which is smaller than the light wavelength in the dielectric, $\lambda_{p}=\lambda / n_{d}$. What makes SPPs special is that the wavelength could be made as small as required, even vanishing for localized surface plasmons. ${ }^{2}$ In our lenslike structure, if we want to improve the focusing resolution, we could decrease $\lambda_{\text {spp }}$. For a fixed vacuum wavelength, the simplest approach to increase $n_{\text {eff }}$ is to make the thickness $h_{0}$ smaller. Figure 5 shows the dependency of FWHM along $x$ at the focal plane for a fixed wavelength $\lambda=0.7 \mu \mathrm{m}$ and $w_{0}=800 \mathrm{~nm}$. Figures 5(a) and 5(b) show the field distribution $|E|$ along propagation for the two points marked in Fig. 5(c) in the $x-z$ plane of $y=h_{0} / 2$
(20 and $10 \mathrm{~nm}$, respectively). The white dashed lines indicate the focal planes. Insets (i) and (ii) show the transverse field distribution of $|E|$ at the two focusing planes. As shown in Fig. 5, the FWHM could be as small as $100 \mathrm{~nm}$. This could be further improved by decreasing $R_{0}$ and/or increasing the focusing strength $\Omega$. Our results clearly show that the light is fully confined in the dielectric along $y$ and trapped in the optical parabolic potential along $x$.

In conclusion, we have suggested the concept of polychromatic plasmonics and demonstrated a broadband plasmonic lens based on a parabolically modulated MDM structure. We have shown the plasmonic lens allows for complete three-dimensional subwavelength control of a beam, bringing a potential functionality for manipulation of ultrashort optical signals. We anticipate the proposed structure is a promising candidate for broadband plasmonic applications including subwavelength white light imaging, polychromatic plasmon solitons, and ultrashort pulse plasmonic nanolasers.

We thank A. A. Sukhorukov, Z. Xu, A. S. Desyatnikov, C. G. Poulton, and A. E. Minovich for useful discussions, and acknowledge support from the Australian Research Council and the National Computational Infrastructure Merit Allocation Scheme.
${ }^{1}$ L. Cao and M. L. Brongersma, Nat. Photonics 3, 12 (2009).

${ }^{2}$ A. V. Zayats, I. I. Smolyaninov, and A. A. Maradudin, Phys. Rep. 408, 131 (2005).

${ }^{3}$ D. K. Gramotnev and S. I. Bozhevolnyi, Nat. Photonics 4, 83 (2010).

${ }^{4}$ B. Lee, S. Kim, H. Kim, and Y. Lim, Prog. Quantum Electron. 34, 47 (2010).

${ }^{5}$ Y. M. Liu, T. Zentgraf, G. Bartal, and X. Zhang, Nano Lett. 10, 1991 (2010).

${ }^{6}$ P. A. Huidobro, M. L. Nesterov, L. Martin-Moreno, and F. J. GarciaVidal, Nano Lett. 10, 1985 (2010).

${ }^{7}$ L. Verslegers, P. B. Catrysse, Z. F. Yu, and S. H. Fan, Phys. Rev. Lett. 103, 033902 (2009).

${ }^{8}$ M. A. Noginov, G. Zhu, M. Mayy, B. A. Ritzo, N. Noginova, and V. A. Podolskiy, Phys. Rev. Lett. 101, 226806 (2008).

${ }^{9}$ M. I. Stockman, Phys. Rev. Lett. 93, 137404 (2004).

${ }^{10}$ A. R. Davoyan, I. V. Shadrivov, A. A. Zharov, D. K. Gramotnev, and Y. S. Kivshar, Phys. Rev. Lett. 105, 116804 (2010).

${ }^{11}$ A. Szameit, I. L. Garanovich, M. Heinrich, A. A. Sukhorukov, F. Dreisow, T. Pertsch, S. Nolte, A. Tuennermann, and Y. S. Kivshar, Nat. Phys 5, 271 (2009).
${ }^{12}$ S. Vedantam, H. Lee, J. Tang, J. Conway, M. Staffaroni, and E. Yablonovitch, Nano Lett. 9, 3447 (2009).

${ }^{13}$ M. Durach, A. Rusina, and M. I. Stockman, Nano Lett. 7, 3145 (2007).

${ }^{14}$ E. Verhagen, M. Spasenović, A. Polman, and L. K. Kuipers, Phys. Rev. Lett. 102, 203904 (2009).

${ }^{15}$ T. Sondergaard, S. I. Bozhevolnyi, S. M. Novikov, J. Beermann, E. Devaux, and T. W. Ebbesen, Nano Lett. 10, 3123 (2010).

${ }^{16}$ H. Gao, J. K. Hyun, M. H. Lee, J.-C. Yang, L. J. Lauhon, and T. W. Odom, Nano Lett. 10, 4111 (2010).

${ }^{17}$ S. I. Bozhevolnyi and J. Jung, Opt. Express 16, 2676 (2008).

${ }^{18}$ S. I. Bozhevolnyi and K. V. Nerkararyan, Opt. Express 17, 10327 (2009).

${ }^{19}$ J. A. Arnaud, Beam and Fiber Optics (Academic Press, New York, 1976).

${ }^{20}$ S. I. Bozhevolnyi, Opt. Express 14, 9467 (2006).

${ }^{21}$ D. Marcuse, Light Transmission Optics (Van Nostrand Reinhold, New York, 1972). 